\title{
The XMM-Newton spectral-fit database
}

\author{
A. Corral $^{1}$, I. Georgantopoulos ${ }^{1}$, S. Rosen ${ }^{2}$, M. G. Watson ${ }^{2}$, \\ K. Page $^{2}$, and G. C. Stewart ${ }^{2}$ \\ ${ }^{1}$ Institute for Astronomy, Astrophysics, Space Applications, and Remote Sensing (IAASARS), \\ National Observatory of Athens (NOA), \\ I.Metaxa \& Vas. Pavlou St., 15236, Penteli, Greece \\ email: acorral@noa.gr \\ ${ }^{2}$ X-ray and Observational Astronomy Group (XROA), Department of Physics and Astronomy, \\ University of Leicester, \\ University Road, Leicester, LE1 7RH, United Kingdom
}

\begin{abstract}
The XMM-Newton spectral-fit database is an ongoing ESA funded project aimed to construct a catalogue of spectral-fitting results for all the sources within the XMM-Newton serendipitous source catalogue for which spectral data products have been pipeline-extracted ( $\gtrsim 120,000 \mathrm{X}$-ray source detections). The fundamental goal of this project is to provide the astronomical community with a tool to construct large and representative samples of X-ray sources by allowing source selection according to spectral properties.
\end{abstract}

Keywords. X-ray surveys.

\section{Introduction}

The XMM-Newton serendipitous source catalogue is the largest catalogue of X-ray sources built to date, see Watson et al. (2009). In its latest version, the 3XMM-Newton Data Release 4 (3XMM DR4 $\dagger$ ), it contains photometric information for more than 500,000 source detections, corresponding to $\sim 370,000$ unique sources. Besides, spectra and time series were also extracted if the number of source counts collected by the EPIC camera was $>100$ counts. The resulting catalogue contains spectra for more than 120,000 detections corresponding to $\sim 85,000$ unique sources. The project described here, is aimed to take advantage of the great wealth of data and information contained within the XMM-Newton source catalogue, to construct a database composed of spectral-fitting results.

\section{Automated spectral-fitting procedure}

The XMM-Newton spectral-fit database is constructed by using automated spectral fits applied to the pipe-line extracted spectra within the 3XMM DR4 catalogue. The software used to perform the spectral fits is XSPEC v12.7 (see Arnaud (1996)). The statistics used to fit the data is Cash statistics, implemented as C-stat in XSPEC. This statistics was selected, to optimise the spectral fitting in the case of low quality spectra. However, even using this statistics, a lower limit on the number of counts in each individual spectrum of 50 counts per instrument had to be imposed to ensure a minimum quality on the spectral fits. Therefore, the spectral-fit database is composed of spectral-fitting results for $\gtrsim 114,000$ detections, corresponding to $\sim 77,000$ unique sources.

$\dagger$ http://xmmssc-www.star.le.ac.uk/Catalogue/3XMM-DR4/ 
Three energy bands are considered during the spectral fits: Full/Total band (0.5 $10 \mathrm{keV})$, Soft band $(0.5-2 \mathrm{keV})$, and Hard band $(2-10 \mathrm{keV})$. Six spectral models have been implemented in the spectral-fitting pipe-line: three simple models (models 1 to 3 ), and three more complex models (models 4 to 6 ). These six models were designed and optimised to reproduce the most commonly observed X-ray spectral shapes among different astronomical sources. The three simple models are applied to all sources provided that the number of counts collected in the energy band in use is $>50$ counts:

1. Absorbed power-law model (wa*pow): A photoelectrically absorbed power-law model. This model is applied in the three energy bands.

2. Absorbed thermal model (wa*mekal): A photoelectrically absorbed thermal model. This model is applied in the total and soft bands.

3. Absorbed black-body model (wa*bb): A photoelectrically absorbed black-body model. This model is only applied in the soft band, and its primary use is to obtain initial input parameters for a more complex model.

The three more complex models are only applied if the number of counts collected in the total band is $>500$ counts $(\gtrsim 28,000$ detections, corresponding to $\sim 18,000$ unique sources):

4. Absorbed power-law model plus thermal model (wa(mekal+wa*pow)): An absorbed thermal model plus a more highly absorbed power-law model.

5. Double power-law model (wa*(pow+wa*pow)):. Two power-law models with different photon indices, and additional absorption for the second power-law component.

6. Black-body plus power-law model (wa*(bb+pow)):. A black-body plus a powerlaw component, both absorbed by the same amount of gas.

\section{Automated spectral-fitting results}

A full description of this project as well as the full spectral-fit database is presented in the webpage of this project:

\section{http://xraygroup.astro.noa.gr/Webpage-prodec/index.html.}

The spectral-fit database contains one row per source and observation, listing source information, and spectral-fit output parameters and errors, as well as fluxes and additional information about the goodness of fit for every model applied. In this way, users can construct large and representative samples of X-ray sources by querying this database according to spectral properties. In addition, and in order to test the reliability of the automated fits, the spectral-fitting process has also been applied to a sample of X-ray sources extracted from the SDSS/XMM-Newton cross-correlation presented in Georgakakis \& Nandra (2011). The spectral-fitting pipe-line was modified to include the effect of redshift and Galactic absorption, in the case of sources with either spectroscopic or photometric redshifts within that sample.

Acknowledgements: A. Corral acknowledges financial support by the European Space

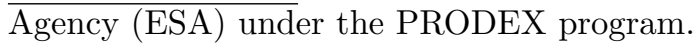

\section{References}

Arnaud K. A. 1996, ASP-CS, 101, 17

Georgakakis A. \& Nandra K. 2011, MNRAS, 414, 992

Watson M. G., et al. 2009, A\&AA, 493, 339 